\title{
Error analysis in Barker's method: extension to ternary systems
}

\author{
I.M.A. Fonseca, L.Q. Lobo * \\ Departamento de Engenharia Química, Universidade de Coimbra, 3000 Coimbra, Portugal \\ Received 1 December 1994; accepted 7 November 1998
}

\begin{abstract}
An extension of Barker's method to ternary systems is briefly outlined. Expressions for the standard deviations of the excess molar Gibbs free energy and the equilibrium pressure as functions of composition are obtained. These expressions are applied to accurate experimental data for the cryogenic (liquid) mixture $\mathrm{CH}_{3} \mathrm{~F}+\mathrm{N}_{2} \mathrm{O}+\mathrm{Xe}$ recently measured in our laboratory. (C) 1999 Elsevier Science B.V. All rights reserved.
\end{abstract}

Keywords: VLE low-pressure; Ternary systems; Errors; Barker's method

\section{Introduction}

In the experimental determination of vapour-liquid equilibrium (VLE) using isothermal static cells the measured properties are the equilibrium pressure $P_{\exp }$ of the mixture and the amount of substance $n_{\mathrm{i}}$ of each component introduced into the cell. Reduction of these data to obtain $P-x-y$ phase diagrams is usually made by using Barker's method [1]. This method has been widely used for binary and ternary systems but in most cases the statistical analysis of the errors associated with it has not been reported. Góral [2] described a procedure to carry out such analysis for binary systems, and Kolasińska et al. [3] applied it assuming the Wilson model for the excess molar Gibbs free energy $G^{\mathrm{E}}$. In this paper we have extended this kind of study to ternary mixtures, and applied it to VLE data for the system $\mathrm{CH}_{3} \mathrm{~F}(1)+\mathrm{N}_{2} \mathrm{O}(2)+\mathrm{Xe}(3)$ and its constituent binaries, at $182.33 \mathrm{~K}$ [4], recently obtained in our laboratory by a modified version of the accurate low-temperature technique introduced and developed by Davies et al. [5] and Fonseca and Lobo [6].

Since the equilibrium pressure is a nonlinear function of the adjustable parameters in the assumed $G^{\mathrm{E}}$ model expression, Barker's method embodies a nonlinear regression technique. Therefore, the

\footnotetext{
${ }^{*}$ Corresponding author. Tel.: +351-39-833407; fax: +351-39-834971. 
uncertainties associated with such parameters are to be obtained from the variance-covariance matrix [7]. Using the propagation law of errors the standard deviations surfaces for $P$ and $G^{\mathrm{E}}$ as functions of composition for ternary systems can be calculated. In this paper we report on such calculations.

\section{Barker's method: outline of application to ternary mixtures}

In essence Barker's method optimizes the parameters in the assumed expression for the liquid composition dependence of $G^{\mathrm{E}}$ through an iterative procedure which minimizes the sum of the squares of the pressure residuals $R_{\mathrm{P}}$,

$$
R_{\mathrm{P}}=P_{\exp }-P \text {. }
$$

The calculated equilibrium pressure $P$ is obtained from

$$
P=\sum_{i} x_{i} \gamma_{i} P_{i} * \beta_{i},
$$

where $x_{i}$ is the mole fraction of component $i$ in the liquid mixture, $\gamma_{i}$ is its activity coefficient, and $\beta_{i}$ is a factor which (mainly) takes into account the vapour-phase non-ideality, usually through a two-term virial equation of state,

$$
\beta_{i}=\exp \left\{\left\{\left(V_{i} *-B_{i i}\right)\left(P-P_{i}^{*}\right)-\frac{1}{2} P \sum_{j} \sum_{k} y_{i} y_{k}\left(2 \delta_{j i}-\delta_{j k}\right)\right\}: R T\right\} .
$$

In these expressions $y_{i}$ represents the mole fraction of $i$ in the vapour, $P_{i}^{*}$ and $V_{i}{ }^{*}$ are the vapour pressure and the liquid molar volume of pure component $i$, respectively, $B_{i i}$ is the second virial coefficient of $i$, and $\delta_{i j}=2 B_{i j}-B_{i i}-B_{j j}$. Initial estimates for the values of the mole fractions in the vapour $y_{i}$ are found by assuming Raoult's law, and in the next iterations by taking

$$
y_{i}=\left(x_{i} y_{i} P_{i}^{*} / P\right) \beta_{i} .
$$

Expressions for the activity coefficients are derived from the rigorous thermodynamic relationship

$$
\ln \gamma_{i}=\left[\partial\left(n^{\mathrm{L}} G^{E}\right) / \partial n_{i}^{\mathrm{L}}\right]_{T, P, n_{j}}
$$

where $n^{\mathrm{L}}=\sum_{i} n_{i}^{\mathrm{L}}$ and the superscript ${ }^{\mathrm{L}}$ refers to the liquid mixture. For ternary systems we have chosen to represent the reduced quantity $g^{\mathrm{E}}\left(=G^{\mathrm{E}} / \mathrm{RT}\right)$ in the form

$$
g^{\mathrm{E}}=\sum_{\text {all binaries }} g_{i j}^{\mathrm{E}}+g_{123}^{\mathrm{E}},
$$

where the binary terms $g_{i j}^{\mathrm{E}}$, and the ternary one $g_{123}^{\mathrm{E}}$ are assumed to be Redlich-Kister functions of the mole fractions in the liquid,

$$
g_{i j}^{E}=x_{i} x_{j}\left\{A_{i j}+B_{i j}\left(x_{i}-x_{j}\right)+C_{i j}\left(x_{i}-x_{j}\right)^{2}\right\},
$$

and

$$
g_{123}^{E}=x_{1} x_{2} x_{3}\left(c_{0}-c_{1} x_{1}-c_{2} x_{2}\right)
$$


In these expressions $A_{i j}, B_{i j}, C_{i j}, c_{0}, c_{1}$ and $c_{2}$ are the adjustable parameters mentioned above. From Eqs. (5) and (6) the activity coefficient of component 1 becomes

$$
\begin{aligned}
\ln \gamma_{1}= & x_{2}\left[2\left(A_{12}+B_{12}+C_{12}\right) x_{1}\left(1-x_{1}\right)+\left(A_{12}-B_{12}+C_{12}\right) x_{2}\left(1-2 x_{1}\right)\right. \\
& \left.+4 C_{12} x_{1} x_{2}\left(3 x_{1}-2\right)\right]+x_{3}\left[2\left(A_{13}+B_{13}+C_{13}\right) x_{1}\left(1-x_{1}\right)+\left(A_{13}-B_{13}+C_{13}\right) x_{13}\right. \\
& \left.\times\left(1-2 x_{1}\right)+4 C_{13} x_{1} x_{3}\left(3 x_{1}-2\right)\right]+x_{2} x_{3}\left[12 C_{23} x_{2} x_{3}-2\left(A_{23}-B_{23}+C_{23}\right) x_{3}\right. \\
& \left.-2\left(A_{23}+B_{23}+C_{23}\right) x_{2}\right]+x_{2} x_{3}\left[c_{0}\left(1-2 x_{1}\right)-c_{1} x_{1}-\left(1-3 x_{1}\right)\left(c_{1} x_{1}+c_{2} x_{2}\right)\right] .
\end{aligned}
$$

Similar expressions for $\ln \gamma_{2}$ and $\ln \gamma_{3}$ are obtained from Eq. (9) by circular change of subscripts. The activity coefficients are functions of the liquid composition and of the parameters present in the $G^{\mathrm{E}}$ model. Values for the parameters $A_{i j}, B_{i j}$ and $C_{i j}$ are arrived at from binary data alone while those for the parameters $c_{0}, c_{1}$ and $c_{2}$ are found by regression of the experimental data for the ternary mixtures, i.e., by solving the matrix equation

$$
[\mathbf{L}][\boldsymbol{\delta} c]=[\mathbf{T}]
$$

where

$$
\begin{aligned}
& {[\mathbf{L}]=\left[\begin{array}{lll}
\sum\left(\frac{\partial P}{\partial c_{0}}\right)^{2} & \sum\left(\frac{\partial P}{\partial c_{1}}\right)\left(\frac{\partial P}{\partial c_{0}}\right) & \Sigma\left(\frac{\partial P}{\partial c_{2}}\right)\left(\frac{\partial P}{\partial c_{0}}\right) \\
\Sigma\left(\frac{\partial P}{\partial c_{0}}\right)\left(\frac{\partial P}{\partial c_{1}}\right) & \Sigma\left(\frac{\partial P}{\partial c_{1}}\right)^{2} & \Sigma\left(\frac{\partial P}{\partial c_{2}}\right)\left(\frac{\partial P}{\partial c_{1}}\right) \\
\Sigma\left(\frac{\partial P}{\partial c_{0}}\right)\left(\frac{\partial P}{\partial c_{2}}\right) & \sum\left(\frac{\partial P}{\partial c_{1}}\right)\left(\frac{\partial P}{\partial c_{2}}\right) & \sum\left(\frac{\partial P}{\partial c_{2}}\right)^{2}
\end{array}\right],} \\
& {[\boldsymbol{\delta} \boldsymbol{c}]=\left[\begin{array}{l}
\delta c_{0} \\
\delta c_{1} \\
\delta c_{2}
\end{array}\right] \text {, and }[\mathbf{T}]=\left[\begin{array}{c}
\sum R_{p}\left(\frac{\partial P}{\partial c_{0}}\right) \\
\sum R_{p}\left(\frac{\partial P}{\partial c_{1}}\right) \\
\sum R_{p}\left(\frac{\partial P}{\partial c_{2}}\right)
\end{array}\right]}
\end{aligned}
$$

All the summations in these matrices are taken over all the $M$ ternary experimental points. The elements of matrix [L] are obtained directly from Eq. (2). The elements $\delta c_{k}(k=0,1,2)$ represent increments in the $c_{k}$ parameters, being obtained from Eq. (10) by inversion:

$$
[\boldsymbol{\delta} c]=[\mathrm{L}]^{-1}[\mathrm{~T}] \text {. }
$$


Table 1

Values of the parameters in Eqs. (6)-(8) with their respective standard deviations [4]

\begin{tabular}{lll}
\hline$A_{12}=0.1248 \pm 0.0057$ & $B_{12}=-0.0862 \pm 0.0034$ & $C_{12}=0.0645 \pm 0.0164$ \\
$A_{13}=1.7470 \pm 0.0018$ & $B_{13}=-0.1467 \pm 0.0039$ & $C_{13}=0.1957 \pm 0.0081$ \\
$A_{23}=1.2126 \pm 0.0148$ & $B_{23}=0.0715 \pm 0.0194$ & $C_{23}=0.0506 \pm 0.0221$ \\
$c_{0}=-0.6711 \pm 0.0746$ & $c_{1}=-0.4088 \pm 0.1354$ & $c_{2}=-0.5585 \pm 0.1488$
\end{tabular}

The iterations start by assuming $c_{0}=c_{1}=c_{2}=0$ (i.e., $g_{123}^{\mathrm{E}}=0$ ), and the process is then recycled with new values for the element matrices $[\mathbf{L}]^{-1}$ and $[\mathbf{T}]$ by taking corrected values of $c_{k}$ given by

$$
c_{k}=c_{k}(\text { last iteration })+\delta c_{k},
$$

until a situation is reached for which the $\delta c_{k}$ become smaller than a pre-defined value (e.g., $\delta c_{k}<10^{-6}$ in this work).

\section{Error analysis} [D],

The calculation of matrix $[\mathbf{L}]^{-1}$ immediately yields [7] the so called variance-covariance matrix

$$
[\mathbf{D}]=[\boldsymbol{L}]^{-1} s_{\mathrm{P}}^{2},
$$

where

$$
s_{\mathrm{P}}^{2}=\sum_{1}^{M} R_{\mathrm{P}}^{2} /(M-m)
$$

represents the variance of the equilibrium pressure, and $m$ is the number of adjustable parameters in Eq. (8). The diagonal elements $D_{i i}$ in the symmetrical matrix [D] are the variances of the parameters. Both $D_{i i}$ and the covariances $D_{i j}$ (i.e., the non-diagonal elements) are affected by the experimental uncertainty incorporated in $s_{\mathrm{p}}^{2}$ as shown by Eq. (14). Three independent variance-covariance matrices for the binary systems are obtained exactly in the same way. They are also independent from that for the ternary system. Indeed each set of binary data (and the ternary one) concerns a different mixture embodying different experimental uncertainties and originating different values of $s_{\mathrm{p}}(15)$.

Table 2

Values of the covariances of the parameters in Eqs. (6)-(8)

\begin{tabular}{lll}
\hline $\operatorname{cov}\left(A_{12}, B_{12}\right)=1.528 \times 10^{-5}$ & $\operatorname{cov}\left(A_{12}, C_{12}\right)=-6.953 \times 10^{-5}$ & $\operatorname{cov}\left(B_{12}, C_{12}\right)=-3.605 \times 10^{-5}$ \\
$\operatorname{cov}\left(A_{13}, B_{13}\right)=3.272 \times 10^{-6}$ & $\operatorname{cov}\left(A_{13}, C_{13}\right)=-2.449 \times 10^{-6}$ & $\operatorname{cov}\left(B_{13}, C_{13}\right)=-1.559 \times 10^{-5}$ \\
$\operatorname{cov}\left(A_{23}, B_{23}\right)=-2.462 \times 10^{-4}$ & $\operatorname{cov}\left(A_{23}, C_{23}\right)=-5.519 \times 10^{-4}$ & $\operatorname{cov}\left(B_{23}, C_{23}\right)=-8.334 \times 10^{-4}$ \\
$\operatorname{cov}\left(c_{0}, c_{1}\right)=-7.401 \times 10^{-3}$ & $\operatorname{cov}\left(c_{0}, c_{2}\right)=8.109 \times 10^{-3}$ & $\operatorname{cov}\left(c_{1}, c_{2}\right)=-4.179 \times 10^{-3}$ \\
\hline
\end{tabular}


The uncertainty associated with any other variable which is itself a function of the optimized parameters mentioned above, namely the $G^{\mathrm{E}}$ function, is readily obtained as follows. Let $f$ be a function of parameters $a, b$, and $c$,

$$
f=F(a, b, c),
$$

whose uncertainties may assume any values between $-\sigma_{k}$ and $+\sigma_{k}(k=a, b, c)$. Then, through the propagation law of errors, the variance $\sigma_{f}^{2}$ is given as [7]

$$
\sigma_{f}^{2}=F_{a}^{2} \sigma_{a}^{2}+F_{b}^{2} \sigma_{b}^{2}+F_{c}^{2} \sigma_{c}^{2}+2 F_{a} F_{b} \operatorname{cov}(a, b)+2 F_{a} F_{c} \operatorname{cov}(a, c)+2 F_{b} F_{c} \operatorname{cov}(b, c)
$$

where the usual convention has been used to indicate the partial derivatives, e.g., $F_{a}=(\partial F / \partial a)_{b, c}$. Expressions similar to Eq. (17) omitting the covariance terms have been used. However, these terms can often make a significant contribution to the estimated value of $\sigma_{f}^{2}$, and therefore should not be neglected [8].

Applying Eq. (17) to $g^{\mathrm{E}}$ defined by Eq. (6), an expression for the standard deviation surface of $G^{\mathrm{E}}$ as a function of the composition of the liquid mixture is obtained:

$$
\begin{aligned}
\sigma_{G^{\mathrm{E}}}= & \left\{( R T ) ^ { 2 } \left\{( x _ { 1 } x _ { 2 } ) ^ { 2 } \left[\sigma_{A_{12}}^{2}+\left(x_{1}-x_{2}\right)^{2} \sigma_{B_{12}}^{2}+\left(x_{1}-x_{2}\right)^{4} \sigma_{C_{12}}^{2}+2\left(x_{1}-x_{2}\right) \operatorname{cov}\left(A_{12}, B_{12}\right)\right.\right.\right. \\
& +2\left(x_{1}-x_{2}\right) \operatorname{cov}\left(A_{12}, C_{12}\right)+2\left(x_{1}-x_{2}\right)^{3} \operatorname{cov}\left(B_{12}, C_{12}\right) \\
& +\ldots\{\text { similar terms for the binaries }(1,3) \text { and }(2,3)\} \ldots+\left(x_{1} x_{2} x_{3}\right)^{2} \times\left[\sigma_{c_{0}}^{2}+\sigma_{c_{1}}^{2} x_{1}^{2}\right. \\
& \left.\left.\left.+\sigma_{c_{2}}^{2} x_{2}^{2}-2 \operatorname{cov}\left(c_{0}, c_{1}\right) x_{1}-2 \operatorname{cov}\left(c_{0}, c_{2}\right) x_{2}+2 \operatorname{cov}\left(c_{1}, c_{2}\right) x_{1} x_{2}\right]\right\}\right\}^{1 / 2} .
\end{aligned}
$$

The total number of parameters whose uncertainties contribute to $\sigma_{G^{\mathrm{E}}}$ is, therefore, twelve: three for each binary mixture, and three for the ternary term.

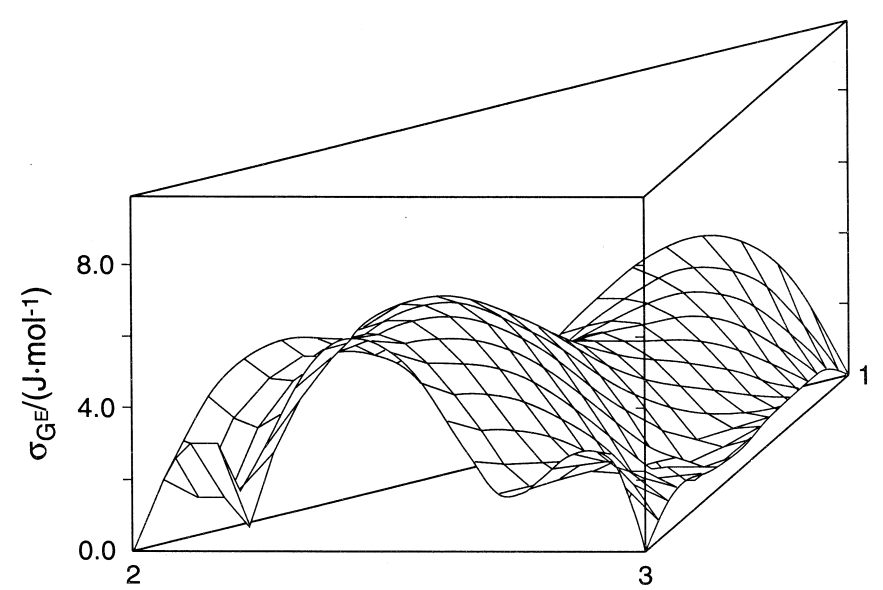

Fig. 1. Perspective view of the standard deviation surface $\sigma_{G^{\mathrm{E}}}$ calculated from Eq. (18), with parameters listed in Tables 1 and 2. $\sigma_{G^{\mathrm{E}}}$ is represented as a function of the mole fractions of the three components. 
In a similar way the standard deviation surface of the equilibrium pressure $\sigma_{\mathrm{P}}$ as a function of the liquid composition is obtained by applying Eq. (17) to $P$ as defined in Eq. (2):

$$
\begin{aligned}
\sigma_{\mathrm{P}}= & \left\{\left(\frac{\partial P}{\partial A_{12}}\right)^{2} \sigma_{A_{12}}^{2}+\left(\frac{\partial P}{\partial B_{12}}\right)^{2} \sigma_{B_{12}}^{2}+\left(\frac{\partial P}{\partial C_{12}}\right)^{2} \sigma_{C_{12}}^{2}+2\left(\frac{\partial P}{\partial A_{12}}\right)\left(\frac{\partial P}{\partial B_{12}}\right) \operatorname{cov}\left(A_{12}, B_{12}\right)\right. \\
& +2\left(\frac{\partial P}{\partial A_{12}}\right)\left(\frac{\partial P}{\partial C_{12}}\right) \operatorname{cov}\left(A_{12}, C_{12}\right)+2\left(\frac{\partial P}{\partial B_{12}}\right)\left(\frac{\partial P}{\partial C_{12}}\right) \operatorname{cov}\left(B_{12}, C_{12}\right) \\
& +\ldots\{\text { similar terms for the binaries }(1,3) \text { and }(2,3)\} \ldots \\
& +\left(\frac{\partial P}{\partial c_{0}}\right)^{2} \sigma_{c_{0}}+\left(\frac{\partial P}{\partial c_{1}}\right)^{2} \sigma_{c_{1}}^{2}+\left(\frac{\partial P}{\partial c_{2}}\right)^{2} \sigma_{c_{2}}^{2}+2\left(\frac{\partial P}{\partial c_{0}}\right)\left(\frac{\partial P}{\partial c_{1}}\right) \operatorname{cov}\left(c_{0}, c_{1}\right)+2\left(\frac{\partial P}{\partial c_{0}}\right) \\
& \left.\times\left(\frac{\partial P}{\partial c_{2}}\right) \operatorname{cov}\left(c_{0}, c_{2}\right)+2\left(\frac{\partial P}{\partial c_{1}}\right)\left(\frac{\partial P}{\partial c_{2}}\right) \operatorname{cov}\left(c_{1}, c_{2}\right)\right\}^{1 / 2} .
\end{aligned}
$$

\section{Results and conclusions}

The values of the parameters in Eq. (6) obtained by Barker's method for $\mathrm{CH}_{3} \mathrm{~F}(1)+\mathrm{N}_{2} \mathrm{O}(2)+\mathrm{Xe}(3)$, at $182.33 \mathrm{~K}$, using experimental data recently obtained in our laboratory [4] are summarized with their respective standard deviations in Table 1. The calculated covariances are listed in Table 2 . The standard deviations surfaces $\sigma_{G}$ and $\sigma_{\mathrm{P}}$ are represented as functions of the liquid mole fractions in Figs. 1 and 2, respectively. Both perspective views in these figures clearly show that the standard deviations in the parameters for the binary mixtures affect the calculated values of $\sigma_{G^{\mathrm{E}}}$ and $\sigma_{\mathrm{P}}$ for

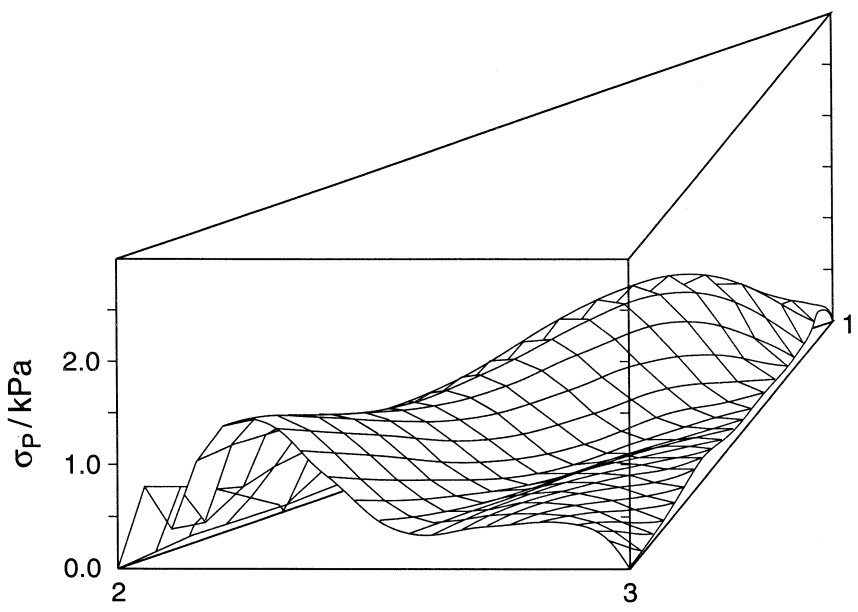

Fig. 2. Perspective view of the standard deviation surface $\sigma_{\mathrm{P}}$ calculated from Eq. (19), with parameters listed in Tables 1 and 2. $\sigma_{\mathrm{P}}$ is represented as a function of the mole fractions of the three components. 
the ternary system. The higher values of these deviations near the $\mathrm{N}_{2} \mathrm{O}(2)+\mathrm{Xe}(3)$ binary region are a consequence of the comparatively much higher values of the parameters standard deviations for this binary mixture. The value of $\sigma_{G^{\mathrm{E}}}$ for the equimolar ternary mixture is about $6 \mathrm{~J} \mathrm{~mol}{ }^{-1}$, half of which arises from $\sigma_{G_{123}^{\mathrm{E}}}$.

\section{References}

[1] J.A. Barker, Aust. J. Chem. 6 (1953) 207-210.

[2] M. Góral, Z. Phys. Chemie, Leipzig 258 (1977) 1040-1044.

[3] G. Kolasińska, P. Oracz, Z. Phys. Chemie, Leipzig 260 (1979) 169-173.

[4] I.M.A. Fonseca, L.Q. Lobo, Fluid Phase Equilibria 113 (1995) 127-138.

[5] R.H. Davies, A.G. Duncan, G. Saville, L.A.K. Staveley, Trans. Faraday Soc. 63 (1967) 855-869.

[6] I.M.A. Fonseca, L.Q. Lobo, Fluid Phase Equilibria 47 (1989) 249-263.

[7] G.E.P. Box, W.E. Hunter, J.S. Hunter, Statistics for Experimenters, Wiley, New York, 1978.

[8] W.E. Wentworth, J. Chem. Educ. 42 (1965) 96-103. 